\title{
Téoros
}

Revue de recherche en tourisme

\section{Le tourisme dans la région du Nord-du-Québec}

Essai sur la structuration de cette industrie à la Baie-James

\section{François Bédard et Hélène Huard}

Volume 18, numéro 2, été 1999

La nordicité

URI : https://id.erudit.org/iderudit/1072132ar

DOI : https://doi.org/10.7202/1072132ar

Aller au sommaire du numéro

Éditeur(s)

Université du Québec à Montréal

ISSN

0712-8657 (imprimé)

1923-2705 (numérique)

Découvrir la revue

Citer cet article

Bédard, F. \& Huard, H. (1999). Le tourisme dans la région du Nord-du-Québec : essai sur la structuration de cette industrie à la Baie-James. Téoros, 18(2),

52-60. https://doi.org/10.7202/1072132ar d'utilisation que vous pouvez consulter en ligne.

https://apropos.erudit.org/fr/usagers/politique-dutilisation/ 


\section{E 5 : 5 : $4: 1$}

\section{LE TOURISME DANS}

\section{LA RÉGION DU Nord-DU-Québec}

ESSAI SUR LA STRUCTURATION DE CETTE INDUSTRIE À LA BAIE-JAMES

\section{François Bédard et Hélène Huard}

La région du Nord-du-Quebec est divisée en deux territoires : le Nunavik, habité principalement par les Inwit, et la Baie-James où cohabitent des populations cries et allochtones ${ }^{2}$. Le Nunavik comprend la partie la plus septentrionale se situant au nord du $55^{\circ}$ parallèle, tandis que la Baie James s'étend entre les $49^{\circ}$ et $55^{\circ}$ paralleles.

La Baie James, territoire sur lequel porte le présent article, couvre une superficie de $333255 \mathrm{~km}^{2}$, soit l'équivalent d'un cinquième dụ Québec, de deux fois et demie l'Angleterre ou encore des deux tiers de la France. Le territoire compte neuf villages cris et dix villes, localités, agglomérations et hameaux allochtones. La faible densité de la population, les conditions de vie liées au climat rigoureux, l'isolement géographique des communautés, l'éloignement des grands centres, les droits territoriaux des autochtones, une organisation sociale, politique et économique distincte pour les autochtones et les allochtones sont autant d'éléments dont la Baie-James doit tenir compte dans la structuration de son industrie du tourisme.

Contrairement aux autres régions du Québec où il existe une association touristique régionale (ATR) depuis une vingtaine d'années, la responsabilité du développement du tourisme dans la région du Nord-du-Québec relevait du Gouvernement québécois jusqu’en 1998, année où Tourisme Québec proposait aux intervenants de la région la création d'une association touristique régionale. Selon les termes de la proposition gouvernementale, la nouvelle ATR serait composée de trois entités distinctes représentant chacune un groupe ethnique présent dans la région : le Conseil régional de développement
Kativik/Association touristique du Nunavik (pour représenter les entreprises touristiques inuit, l'Administration régionale crie (pour les entreprises cries), et le Conseil régional de développement de la Baie James ${ }^{3}$, Tourisme Baic-James (pour les entreprises allochtones). Au moment de la rédaction de cet article, l'Association touristique du Nunavik et Tourisme Baie-James avaient signé l'entente avec Tourisme Québec, alors que les négociations se poursuivaient toujours avec les représentants de la nation crie.

S'inscrivant dans les activités de recherche qui découlent de deux ententes de partenariat, une entre l'UQAM et la Municipalité de la Baie James et l'autre avec Tourisme Baie-James, la présente contribution vise à apporter un éclairage théorique en regard des efforts de structuration du tourisme en cours à la Baie James, de manière à aider les autorités concernées dans leur prise de décisions.

\section{MÉTHODOLOGIE}

Le modèle des grappes industrielles mis au point par le professeur Michael E. Porter de la Harvard Business School sert d'outil d'analyse de l'industrie du tourisme à la Baie-James.
Les grappes industrielles sont des groupes d'entreprises qui appartiennent à une même branche d'activités, partagent des défis et des exigences communes en matière de développement et généralement rendues hautement interdépendantes par des liens de complémentarité en regard de leurs marchés et de leurs facteurs de production. Le schéma 1 représente le modèle de grappe industrielle adapté en 1993 à 1'industrie du tourisme au Québec par' un groupe de travail du gouvernement québécois ${ }^{4}$. Le tourisme repose sur l'interaction et l'intégration d'un ensemble d'industries organisées en fonction d'un but commun : la satisfaction du touriste-consommateur et, de ce fait, la rentabilisation des entreprises touristiques. Ces industries - transport, hébergement, restauration, divertissement et loisir - établissent des echanges avec l'industrie noyau (le tourisme) à titre de fournisseurs ou d'acheteurs. Le modèle met en évidence les intrants (les composantes) du produit touristique, son mode de production et de distribution ainsi que les relations acheteurs-vendeurs entre les éléments de la grappe. Selon le modèle de la grappe industrielle, la compétitivité du produit touristique dépendra surtout de la qualité et de la diversité des intrants du produit.

Pour que le tourisme se développe, il doit d'abord exister une force d'attrait. Celleci réside habituellement dans les ressources naturelles et culturelles ainsi que dans la population d'une région. La région doit posséder des infrastructures d'accès pour permettre au touriste de se rendre à destination. Le touriste a aussi besoin de services, c'est-à-dire de se loger, se nourrir, se divertir et se transporter d'un lieu à l'autre lors de son séjour. Le touriste peut 
composer lui-même son voyage en utilisant les éléments de services dont il a besoin ou il peut acheter un forfait auprès d'un fournisseur de services ou d'un intermédiaire spécialisé en voyages. Les distributeurs font connaittre la destination et rendent le produit accessible aux touristes. Finalement, pour se développer, les entreprises touristiques ont besoin de services complémentaires de coordination, de recherche, de ressources humaines, etc. La grappe industrielle du tourisme doit être complète et un lien doit exister entre tous les éléments de la grappe pour que le voyage-personne se réalise dans des conditions optimales. Les liens prépondérants sont ceux qui doivent exister d'abord entre les fournisseurs de services à la clientèle et les producteurs à qui ils vendent leurs services pour des fins de conception de produits touristiques et, ensuite, avec les distributeurs, directement ou par l'entre-

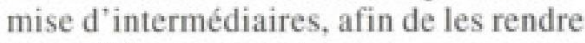
accessibles aux touristes.

Selon le modele, le concept de produit touristique se définit par la combinaison de tous les élements du service que les touristes consomment à partir du moment où ils quittent leur domicile jusqu'à leur retour. Au début des années 1990, Tou= risme Québec a introduit la notion de produits génériques qui se fondait sur l'agrégation de nombreux voyages-personne présentant des caractéristiques similaires quant à la destination, le but ou le comportement de voyage. Un produit générique est un segment de marché auquel correspond une offre de services. Les produits génériques identifiés par Tourisme Québec sont : les séjours urbains, 1es circuits, les séjours de villégiature, le ski alpin, la motoneige, la chasse et la pêche, le tourisme d'aventure et de grande nature ainsi que les congrès, les réunions d'affaires et les voyages de motivation.

Dans sa politique de développement rendue publique en 1998, Tourisme Québec entend faire progressivement référence aux produits génériques en termes d'expériences touristiques. Tourisme Québec considère que le passage de la notion de produit touristique à un concept d'expérience intégrant les produits et les activités répond aux nouvelles tendances observées chez les clientèles et au degré de maturité atteint par l'industrie du tourisme au Québec. Le concept d'expérience se rapproche davantage de celui de produit identifié dans la grappe industrielle du tourisme. lequel comporte une combinaison de tous les éléments des services consommés par les touristes durant leur voyage.

\section{COMPOSANTES DE L'INDUSTRIE DU TOURISME DE LA BAIE-JAMES}

Comme l'illustre le schéma 1, la grappe industrielle du tourisme comprend les composantes suivantes : les ressources, les fournisseurs de services à la clientèle, les producteurs, les distributeurs, le produit et les infrastructures d'accès. Les lignes qui suivent décrivent chacune des composantes en regard de la situation observée a la Baie-James.

\section{LES RESSOURCES}

Les ressources regroupent l'ensemble des cléments du patrimoine naturel et culturel d'une région, auxquels il convient d'ajouter la population locale. Ces ressources représentent souvent l'élé́ment déclencheur et le but principal de voyage des touristes. Elles constituent donc la matière première de l'industrie du tourisme.

Vaste pays de lacs, de rivieres et d'attraits naturels exceptionnels, le territoire de la Baie James est partagé entre les populations cries et allochtones qui l'habitent. Avec la signature de la Convention de la Baie James et du Nord québécois en novembre 1975, les Cris et les Inuit cédaient au gouvernement du Québec leurs droits ancestraux sur les terres du territoire nordique en contrepartie de paiements compensatoires et de divers engagements des gouvernements provincial et fédéral en matière de protection de l'environnement, de développement économique et social et la dévolution de pouvoirs, ce dernier facteur étant l'un des plus importants.

La Convention déterminait l'utilisation des terres, regroupées en trois catégories:

Catégorie I, soit $14025 \mathrm{~km}^{2}$ du territoire de la Baie James et du Nunavik: les villages cris, inuit et naskapis et leur environnement immédiat. Ces terres sont à l'usage exclusif des autochtones.

Catégorie II, soit $162324 \mathrm{~km}^{2}$ : des terres publiques dans le prolongement des ter- res de catégorie I. A l'usage exclusif des autochtones pour les activités de chasse. de pêche et de piégeage, ce sont les autochtones qui autorisent les non-autochtones à y pêcher ou à y chasser.

Catégorie III, soit $889650 \mathrm{~km}^{2}$ : des terres publiques ouvertes à tout le monde. Les autochtones y pratiquent leurs activités traditionnelles à longueur d'annéc et sans limite de prises, dans la mesure où les ressources sont suffisantes. Les autres doivent se conformer aux règlements en vigueur : permis de chasse et de peche obligatoires, de même que respect des dates fixées pour la pratique de ces activités et du nombre de prises établi par la loi.

Les communautés sont regroupées dans plusieurs villes, localités, agglomerations, hameaux et villages enclavés à l'intérieur des limites de la municipalité de la Baie James. La Municipalité a juridiction sur l'ensemble du territoire, à l'exception des terres de catégories I et II, des villes autonomes de Chibougamau, Chapais, Lebel-sur-Quévillon et Matagami et des neuf villages eris: les villages cottiers de Chisasibi, Wemindji, Eastmain ${ }^{6}$, Waskaganish et Whapmagoostui/Kuujuarapik ${ }^{7}$ (situé au-delà du $55^{\circ}$ parallèle), ainsi que les villages à l"intérieur des terres, soit Mistissini, Waswanipi, Nemiscau et OujéBougoumou. Les localités de Beaucanton et de Radisson, les agglomérations de Villebois et de Val-Paradis, ainsi que les hameaux de Miquelon et de Desmaraisville sont sous la juridiction de la municipalité de la Baie James.

La Baie-James compte environ 33000 habitants, dont 20000 allochtones, presque tous francophones, et 13000 cris parlant leur langue maternelle et l'anglais comme langue seconde. ${ }^{8}$ La présence des allochtones est le résultat du développement des industries minières et forestieres, ainsi que du développement du potentiel hydroélectrique de la région. Les Cris ont, pendant plusieurs milliers d'années, vécu en nomades sur le territoire de la Baie James. Au cours du vingtième siècle, ils sont devenus sédentaires à la suite de l'implantation des programmes gouvernementaux comme, par exemple, les programmes scolaires obligatoires.

La population se démarque de la moyenne québécoise par une forte proportion de jeunes et une population âgée très peu élevée. Le groupe des 24 ans et moins 
représente $42,4 \%$ de l'ensemble de la population du territoire alors qu'il constitue $33 \%$ de celle du Québec. Les 65 ans et plus regroupent $2,6 \%$ de la population régionale par rapport a $11,2 \%$ pour la moyenne québécoise, La population des villages cris a augmenté de $29 \%$ de 1981 à 1991 alors que du côté allochtone, le nombre d'habitants fluctue selon l'offre d'emploi. " Avant 1986, l'accessibilité du territoire de la Baie James était limitée aux travailleurs des chantiers de construction du complexe hydroélectrique La Grande et des grandes industries minières et forestières. Les travailleurs de la construction et leur famille venaient s"y établir pour la durée de leur emploi. Certains faisaient et certains font toujours - la commutation aérienne périodique entre leur lieu de travail et leur domicile permanent, situé dans une autre région du Québec, sur la base de huit jours de travail suivis de six jours de congé. Cette population non résidante provenait principalement des régions limitrophes, I $^{*}$ Abitibi-Témiscamingue et le Saguenay-Lac-Saint-Jean. Depuis l'ouverture des routes du territoire au public, de plus en plus de travailleurs miniers et forestiers, ainsi que du personnel administratif, choisissent de s'installer dans la région et de devenir propriétaires de leur demeure.

Le secteur économique autochtone, qui reposait traditionnellement sur la pratique de la chasse et de la peche, se caractérise par de nouvelles activités compatibles aveo les activités traditionnelles - les pourvoiries, entre autres - I la construction d'habitations et d'infrastructures publiques, la création de nouvelles bases économiques cries grâce à l'augmentation de l'activité économique sur le territoire et aux compensations financières qui leur ont été versées dans le cadre de la Convention.

Les économies des municipalités et des localités allochtones sont fragiles et reposent sur la présence du bassin de population nécessaire pour assurer la viabilité des entreprises de services, d'extraction de ressources ou les services gouvernementaux. Les activités f́conomiques s'appuient essentiellement sur l'exploitation des ressources minières et forestières ainsi que sur l'exploitation des ressources hydroélectriques, facteur économique le plus puissant sur le territoire. Les Cris jouent un rôle dans l'économie des municipalités allochtones: ils y font une partie importante de leurs achats et de leurs investissements. Bien que le développement économique lie de plus en plus les deux populations

\section{SCHÉMA 1}

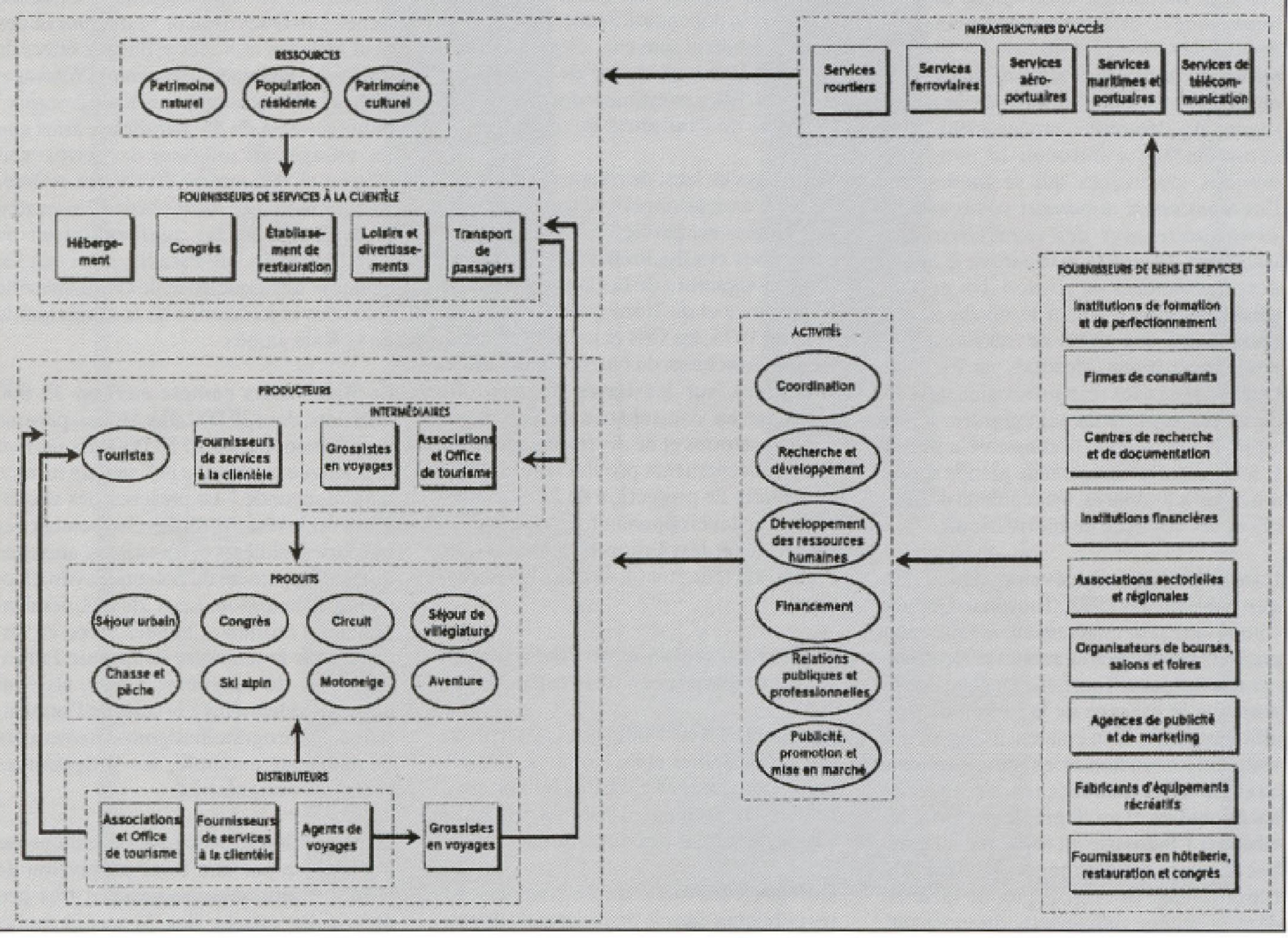


résidantes, la communication et lá concertation demeurent difficiles entre les diverses autorités qui gèrent le territoire, à cause de l'isolement des communautés et des différences entre les groupes ethniques (appartenance à deux groupes linguistiques distincts, stade de développement inégal et aspirations diverses).

\section{LES FOURNISSEURS DE SERVICES À LA CLIENTËLE}

En tourisme, les fournisseurs de services à la clientèle cherchent à exploiter les ressources dans l'intérêt de leur clientèle. Ces entreprises constituent le cour de l'industrie du tourisme et créent la majorité des emplois du secteur. Elles fournissent l'une ou l'autre - et parfois plusieurs des cinq composantes de base d'un produit touristique, soit l'hébergement, les congrès, la restauration, les loisirs et le divertissement ainsi que le transport de passagers. Il est à noter que la plupart des fournisseurs de services à la clientèle jouent également le rôle de producteurs et de distributeurs. Ils investissent temps et argent pour élaborer un produit ou un forfait qui engagera, entre autres, leurs propres entreprises et ils investissent dans des activites de promotion et de vente directe de leur produit.

Les entreprises touristiques de la BaieJames se sont développées autour des ressources dispersées sur le territoire. Du côté allochtone, on dénombre six terrains de camping aménagés, avec une capacité d'accueil de 282 places, ainsi qu'un parc hôtelier de 600 chambres, auquel s'ajoutent une centaine d'unités de relais routiers et les infrastructures d'Hydro-Québec, telles que les résidences où logent ses travailleurs. Dans la plupart des établissements d'hébergement, des services complets de restauration sont offerts. Les autres établissements de restauration sont peu nombreux et peu diversifies. Vingt-sept pourvoiries desservent le territoire ; certaines sont détenues à $100 \%$ par des allochtones et d'autres en partenariat avec des autochtones ${ }^{10}$. Plusieurs localités comptent des clubs de motoneige bien organisés qui s'occupent de l'entretien des sentiers et supervisent la pratique de cette activité. Même si le réseau des pistes de motoneige est bien développé, diversifié et relié aux autres régions du Québec, le lien avec les produits touristiques, les

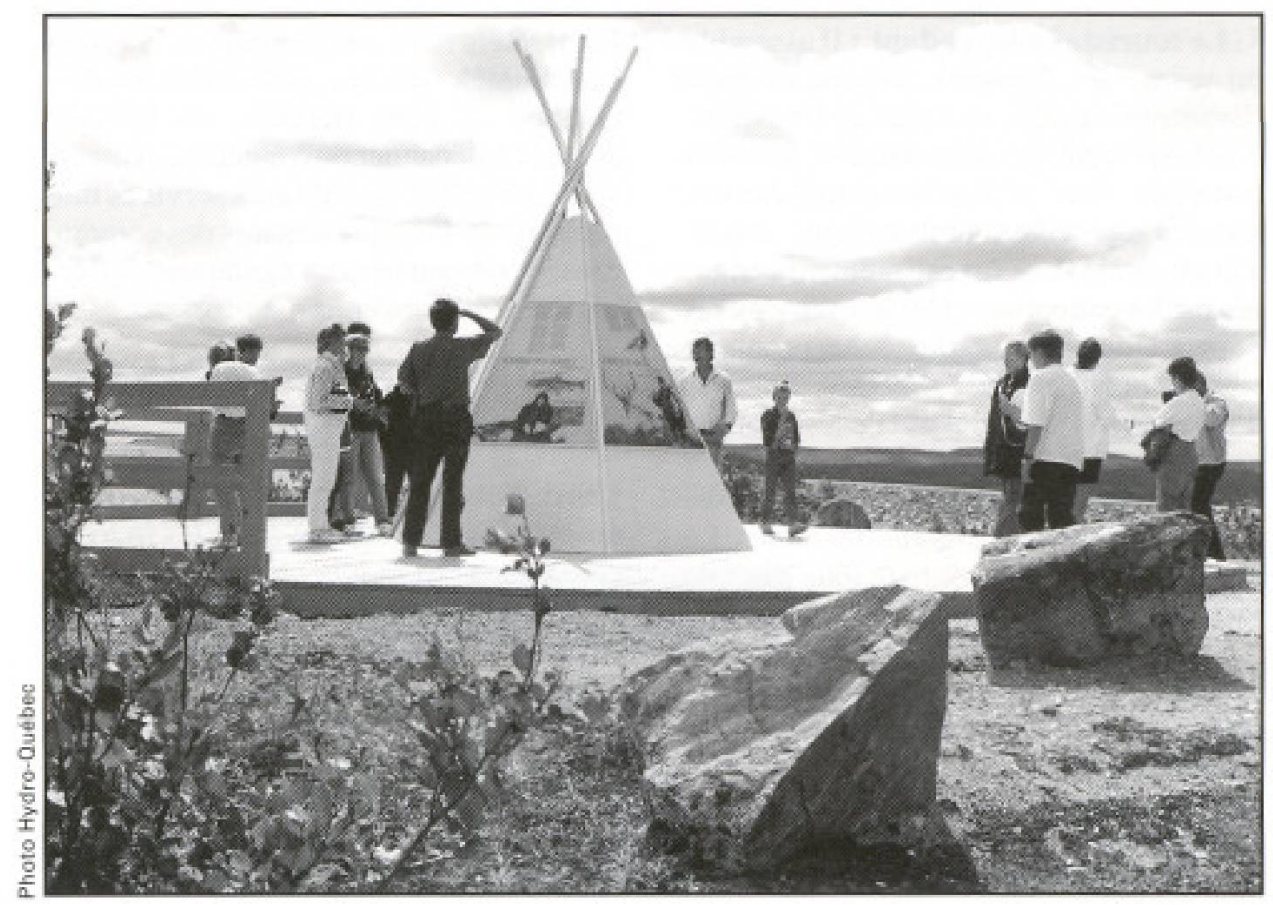

attraits et les infrastructures de services n'est pas fait. Les municipalités interviennent au niveau de l'urbanisme, de l'amenagement du territoire, de la sécurité publique, des équipements culturels et de loisirs. Elles exploitent des équipements de soutien, tels des bureaux d'information touristique, des musées ou des centres d'interprétation, des parcs, des haltes routières et des centres récréatifs.

En ce qui a trait aux entreprises touristi= ques autochtones, le guide \& Le Québec autochtone $x^{11}$ mentionne deux établissements de restauration, huit hôtels, dont certains offrent des services de restauration, une dizaine de pourvoiries et plusieurs boutiques d'artisanat. Il faut toutefois souligner la disparité de la distribution géographique de ces équipements : la majorité sont concentrés dans la section sud du territoire contiguę à la région de l'Abitibi, quelques-uns sont situés près de Radisson et les autres aux alentours du réservoir de la Caniapiscau.

\section{LES PRODUCTEURS}

Les producteurs sont les entreprises, les organismes et les individus qui ont en commun la conception des produits touristiques, résultat d'une combinaison ou d'un montage particulier des intrants que représentent les ressources, les infrastruc- tures et les fournisseurs de services à la clientèle. Il sont, par conséquent, les acheteurs des prestations et des produits touristiques auxquels ils ajoutent une valeur en constituant des forfaits.

Le schéma 1 identifie quatre groupes de producteurs :

1) Les grossistes/voyagistes : Ceux-ci achètent - ou réservent dans le cadre de négociations - les services des fournisseurs de services à la clientèle : blocs de chambres d'hôtel, sièges d'avion, etc.

2) Les associations et les offices de tourisme : Il est peu fréquent que ces demiers remplissent les fonctions de producteurs. Lorsque c'est le cas, la dynamique acheteur-vendeur est assez différente de celle des grossistes/voyagistes, étant donné qu'il s'agit d'organismes à but non lucratif qui agissent généralement à titre de mandataire des fournisseurs de services à la clientèle.

3) Les fournisseurs de services à la clientèle : Ceux-ci font fréquemment office de producteurs, par exemple en élaborant euxmêmes leurs forfaits, en négociant avec des partenaires dans le cadre d'une offre de services conjointe ou, d'une façon générale, en cherchant à intégrer le plus de composantes possible d'un produit touristique complet (ressources, hebergement, restauration, loisirs et divertissement, transport). 
4) Le touriste indépendant : Il assemble lui-même les éléments du produit qu'il consommera : des services de transport, d'hébergement, de restauration et de loisirs et divertissements. Il achète à la pièce des services auprès des fournisseurs de services à la clientèle. Les investissements engagés par les entreprises et les gouvernements pour l'aider dans cette tâche prennent surtout la forme de guides, de services d'information touristique et de réservation.

L'industrie du tourisme comporte une particularitê qui la distingue des autres. La plupart du temps, le touriste joue à la fois le double rôle de client et de producteur. Ainsi, le touriste indépendant représente le principal producteur de produits touristiques à la Baie James : il assemble luimême les différents éléments de son voyage sans recourir à une entreprise. Les résultats d'une enquête sur les services et les amenagements offerts sur le territoire de la Baie James, réalisée de juillet au début septembre 1998 (à l'extérieur de la saison de la chasse et de la pêche), permettent d'esquisser un portrait-type de cette clientèle qui fréquente le territoire à cette période de l'année. ${ }^{12}$ Près de $90 \%$ des touristes sont québécois, $5 \%$ proviennent de l'Ontario et $5 \%$ des États-Unis et de l'Europe ; 65 \% sont âgés de 45 ans et plus, dont le tiers sont des retraités. La majorité des visiteurs ont complété des études collégiales ou universitaires. Les touristes voyagent principalement en couple. Un peu plus de $90 \%$ font un voyage d'agrément d'une durée moyenne de 4,5 jours. La majorité séjourne dans des campings aménagés et voyage en automobile ou en camionnette, alors que le quart voyage en roulotte motorisée. Parmi les visiteurs interrogés, 80 \% en étaient à leur première visite dans la région. Interrogés sur leur satisfaction en regard du séjour, $32 \%$ des visiteurs ont mentionné être satisfaits alors que $65 \%$ étaient très satisfaits. La presque totalité d'entre eux ont jugé que leurs contacts avec les populations locales ont été agréables et $56 \%$ ont mentionné qu'il est probable ou très probable qu'ils reviennent sur le territoire.

Du côté des entreprises, deux agences de voyages réceptives opèrent sur le territoire allochtone et deux autres sur le territoire autochtone. Tourisme Baie-James et la municipalité de la Baie James, qui comptent également des professionnels à temps complet dans le domaine du tourisme. s'ajoutent au nombre des producteurs.
Finalement, des fournisseurs de services à la clientèle, tels les pourvoiries, HydroQuébec - cette dernière est souvent appelée à dépanner ou à aider les visiteurs sur le territoire - certains services municipaux et des représentants des communautés cries participent également, à l'occasion, à la conception de forfaits.

\section{LES DISTRIBUTEURS}

Les distributeurs regroupent les grossistes et les agences de voyages, les fournisseurs de services à la clientèle et les associations et offices de tourisme, dans leurs fonctions de promotion et de vente de produits touristiques. Les distributeurs certains sont du Québec, d'autres de l'extérieur de la province - sont les intermédiaires entre les producteurs et les consommateurs. Leur travail est généralement rémunéré par les producteurs. Les coûts, pour les producteurs, se présentent sous forme de commissions, de frais d'association, de marketing, etc.

Avant la mise sur pied de l'ATR du Norddu-Québec, Tourisme Québec assumait les responsabilités qui échoient habituellement à une association touristique régionale. Cette responsabilitê est maintenant assumée par Tourisme Baie-James pour le territoire allochtone de la Baie James. Tel que mentionné précédemment, les négociations entre Tourisme Québec et les Cris en regard de leur participation à l'ATR du Nord-du-Québec se poursuivent toujours. S'appuyant sur une clause de la Convention de la Baie James et du Nord québécois qui prévoit la création d'une association crie de pourvoirie et de tourisme, les Cris pourraient préférer cette option à celle de devenir une entité constituante de la nouvelle ATR.

Tourisme Québec conserve néanmoins un rôle actif en favorisant une concertation des actions entre les différents intervenants touristiques. À titre d'exemple, Tourisme Québec apporte un appui en matière de commercialisation, particulièrement sur les marchés internationaux ; il agit comme agent de liaison des intérêts du tourisme autochtone du Québec en assurant une représentation au sein de différents comités nationaux, dont celui sur le tourisme autochtone de la Commission canadienne du tourisme, ainsi qu'au sein de l'Aboriginal Tourism Team Canada, un organisme créé en 1998.

\section{LE PRODUIT}

Selon le modèle de la grappe industrielle, le produit touristique est défini par la combinaison de tous les éléments du service que les touristes consomment à partir du moment où ils quittent leur domicile jusqu'à leur retour. Il est en fait le résultat d'un montage ou d'un assemblage particulier d'intrants.

La chasse et la pêche attirent des visiteurs sur le territoire de la Baie James depuis de nombreuses années. Un important réseau de pourvoiries s'est développé autour de ces activités. La clientèle des pourvoiries provient majoritairement de l'extérieur du Québec $(60 \% \text { en } 1995)^{13}$. La visite des centrales hydroélectriques est maintenant le principal produit d'appel de la BaieJames (12 000 visiteurs en 1997) ${ }^{14}$. L'enquête réalisée en 1998 indique que, durant leur séjour, $77 \%$ des touristes ont visité des installations hydroélectriques, $48 \%$ ont fait de la pêche et $44 \%$ ont visité des communautés autochtones. Les autres activités pratiquées par les visiteurs comprennent la découverte du paysage, la visite de musées et de lieux historiques.

La motoneige est une activité qui attire un bon nombre de visiteurs sur les $1000 \mathrm{~km}$ de sentiers fédérés et les milliers de kilomètres de sentiers non balisés de la région. Un vaste réseau de routes forestières permettent aussi aux visiteurs de pratiquer des activités sportives telles que le quad - connu également sous le nom de VTT - le ski de fond, le vélo de montagne, la moto-cross, la randonnée, etc. Des Evénements touristiques se déroulent tout au cours de l'année. Mentionnons, à titre d'exemples, le Raid des Conquérants, le Challenge du Kanada, la Classique internationale de canot de Chibougamau, le Rallye international de motoneige et la Traversée de la Baie James en skis de fond. Plusieurs activités et forfaits sont offerts par les autochtones : motoneige, raquette, observation de la faune et de la flore, excursions en canot de mer, expéditions de chasse et de pêche, safari-photos, etc. Ces activités sont presque exclusivement pratiquées dans le sud du territoire.

Selon les données compilées par la municipalité de la Baie James, un peu plus de 22000 touristes ont emprunté la route de la Baie James en 1998. La fréquentation touristique de la région est plus élevée durant la saison estivale (14 000 person- 
nes). La saison de la chasse au caribou a attiré 8346 personnes de novembre 1997 à fevrier 1998.

Sur les 12000 personnes accueillies par Hydro-Québec en 1997, principalement à la Centrale Robert-Bourassa, $47 \%$ faisaient partie du grand public, $25 \%$, de groupes scolaires, $13 \%$ étaient des visiteurs d'affaires et $10 \%$ étaient venus avec des groupes organisés.

\section{LES INFRASTRUCTURES D'ACCẺS}

Les infrastructures d'accès sont l'ensemble des installations et des équipements permettant d'accéder aux ressources et aux fournisseurs de services à la clientèle. Elles servent un grand nombre d'industries et elles jouent un rôle essentiel dans le développement économique et touristique d'une région.

Le territoire de la Baie James est aujour$\mathrm{d}^{*}$ hui doté d'un réseau routier de qualité. C'est la Société de développement de la Baie James, société para-gouvernementale, qui a supervisé la construction de la route de la Baie James au eout de 450 millions de dollars dans les années 1970 . Cette route traverse le territoire du sud au nord, relie Matagami à Radisson et au complexe La Grande-2 $(620 \mathrm{~km})$ et bifurque vers l'ouest pour rejoindre Chisasibi (100 km). A cet axe nord-sud s'ajoute la route transtaïga qui permet de traverser le nord du territoire d'ouest en est jusqu'au réservoir Caniapiscau. La route du Nord, plus au sud, crée un lien entre Chibougamau et la route de la Baie James, via Némiscau. Quelques routes secondaires s"ajoutent à ces principaux axes routiers. On note l'absence $\mathrm{d}^{\dagger}$ un lien est-ouest direct entre Chibougamau et Matagami dans la partie sud du territoire.

L'ouverture au public de la route de la Baie James en 1986 a favorisé l'essor du tourisme. A la clientèle traditionnelle de chasse et de peche, qui se concentrait pour l'essentiel dans le sud du territoire, se sont ajoutés les visiteurs des centrales hydroélectriques et des villages autochtones ainsi que les amateurs de grande nature.

La région possède aussi des accès aériens de qualité qui permettent un lien avec les autres régions du Québec. Toutes les com= munautés allochtones et autochtones possèdent des services aćroportuaires. Des vols réguliers s"effectuent vers Québec,

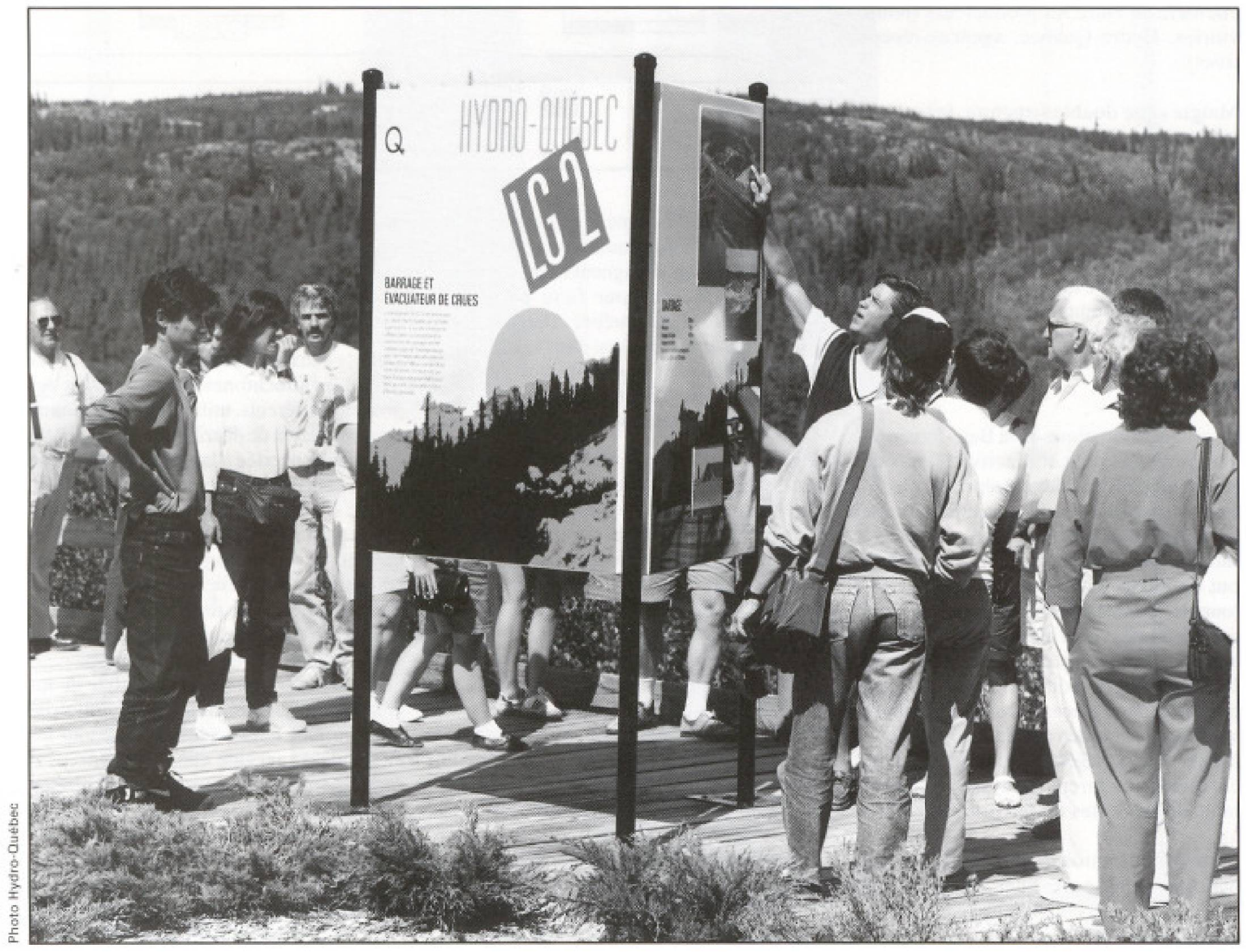


Montréal et le territoire du Nunavik. En outre, des services de nolisement d'avion. d'hélicoptère et d'hydravion sont offerts. La vasté étendue du territoire de la Baie James, son éloignement de la capitale et de la métropole et le petit nombre de passagers expliquent les coûts élevés des transports.

\section{ANALYSE}

L'examen des composantes de l'industrie du tourisme de la Baie James fait ressortir l'existence de deux grappes industrielles du tourisme - l'une allochtone et l'autre autochtone - evoluant en parallèle. Le schema 2 illustre le fonctionnement du tourisme à la Baie James tel qu'observé a. l'hiver 1999. Les liens entre les entreprises touristiques allochtones et autochtones sont encore peu développés, que ce soit entre les fournisseur's de services à la clientèle ou entre les producteurs (pourvoiries, Hydro-Québec, agences réceptives),

Malgré cette double structure, le touriste indépendant, désigné également de touriste producteur, voit le territoire de la Baie James comme un tout. Il prépare son expérience de voyage en choisissant les éléments qui l'intéressent à partir de l'ensemble de l'offre touristique portée à sa connaissance. De façon générale, il n'a pas à prendre en compte les frontières géographiques, ni les considérations juridiques, ethniques, administratives ou politiques locales.

Le produit touristique de la Baie-James est composé d'attraits et d'activités, mis en valeur et gérés par des entreprises allochtones ou autochtones. La concertation entre les entreprises des deux réseaux visant à intégrer des éléments de services dans le but de mieux répondre aux besoins des touristes durant leur séjour sur le territoire est minimale, voire inexistante. Pourtant, aux yeux des touristes, ces éléments sont liés. Ils utilisent indifféremment les équipements mis en place par les municipalités, visitent les grandes centrales hydroélectriques et les villages autochtones, pechent, chassent et admirent le paysage en circulant sur les routes du territoire.

Pour la distribution du produit, deux scénarios peuvent se présenter à court terme : soit que les Cris créent leur propre association de pourvoirie et de tourisme et que
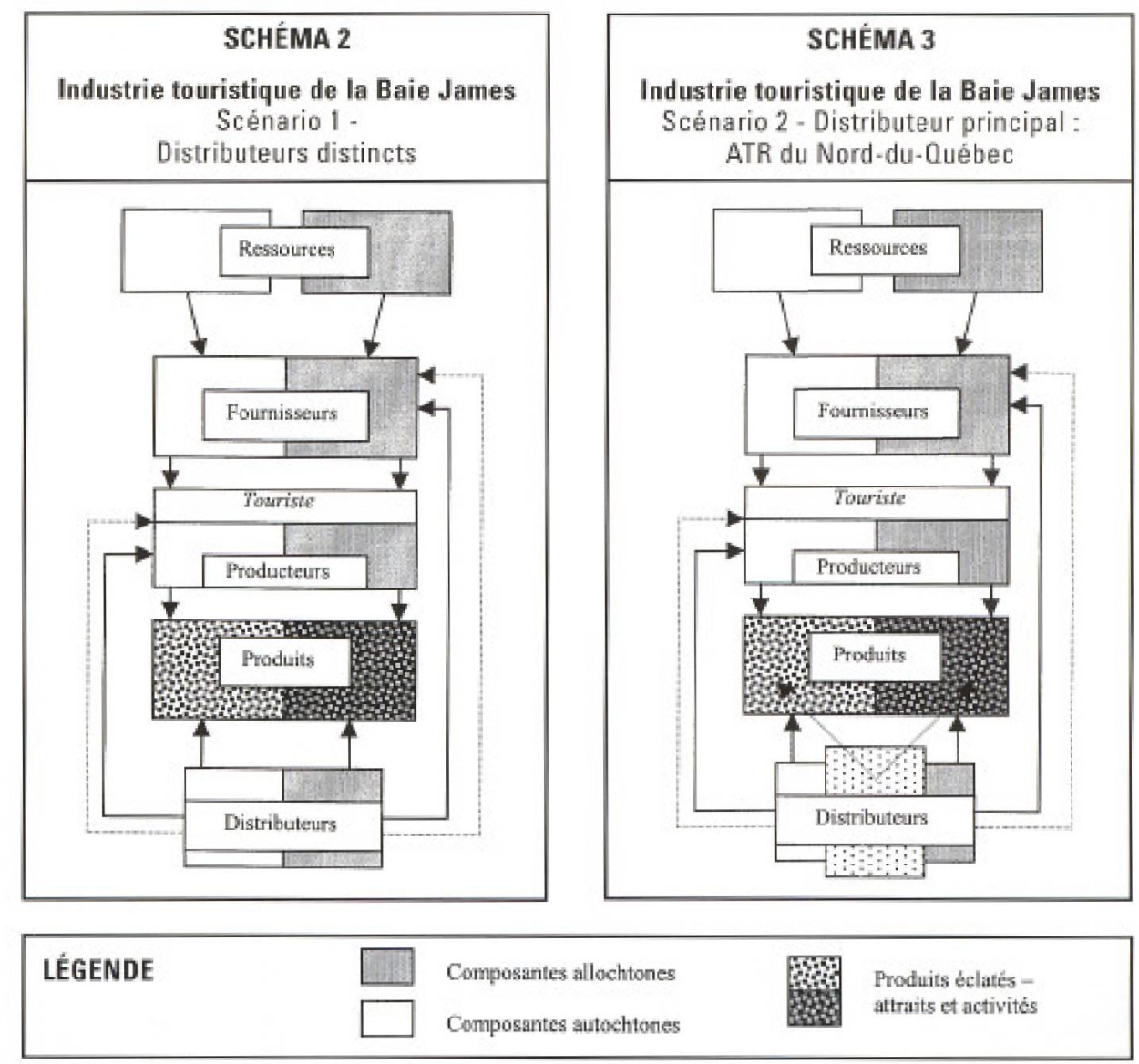

cet organisme devienne le principal agent de distribution du produit autochtone de la region, soit qu'ils signent l'entente avec Tourisme Québec pour faire partie de l'ATR du Nord-du-Québec. Dans ce dernier cas, ils s'inscriraient dans le cadre de développement et de distribution du produit proposé par Tourisme Québec dans sa politique de développement du tourisme.

Examinons l'effet de chacun de ces scénarios sur la structure du tourisme de la Baie-James. Dans le premier scénario (schéma 2), les Cris ne signent pas l'entente avec Tourisme Québec et créent leur propre association de pourvoirie et de tourisme sans lien avec Tourisme Baie-James. La structure parallèle existante est ainsi maintenue. Le touriste indépendant continue à * s approvisionner $*$ auprès des deux groupes de fournisseurs de services à la clientèle ${ }^{15}$, même s'il perç̣oit la Baie James comme une seule destination géographique comportant des attraits naturels et culturels et des activités qui l'intéressent. Un tel scénario implique des coûts de distribution du produit touristique plus élevés ${ }^{16}$ que le second, étant donné que Tourisme Baie-James et les Cris utilisent des stratégies et des moyens qui leur sont propres pour joindre la clientèle. Une telle situation rend très arcu et coûteux le travail de positionnement et de distribution de la Baie-James comme destination touristique.

Dans le deuxième scénario (schéma 3), les Cris et les allochtones, tout en offrant des produits différents, utilisent l'ATR comme principal agent de distribution et travaillent de façon concertée à la commercialisation et à la distribution du produit touristique. Même si ce scénario présente une amélioration par rapport à celui décrit précédemment, il ne rencontre pas, lui non plus, les conditions essentielles du modèle théorique de la grappe du tourisme. Le modèle, rappelons-le, stipule qu' un lien doit exister entre tous les éléments de la grappe pour que le voyage-personne se réalise de façon optimale, et définit le produit touristique comme une combinaison de tous les eléments du service que le touriste consomme à partir du moment où il quitte son domicile jusqu'à son retour. Puisque le touriste indépendant et le produit touristique sont étroitement liés, les structures touristiques parallèles observées présentement à la Baie-James auraient avantage à être intégrées tel qu'illustrế au schéma 4 . 
Les ressources propres aux Cris et aux allochtones, utilisées par les fournisseurs de services à la clientèle, demeurent différenciées car elles constituent le fondement de la personnalité distinctive de la Baie-James. Cependant, la mise sur pied de partenariats pour le développement du produit et sa mise en marché permet de maximiser les investissements consacrés à ces activités. Les fournisseurs de services peuvent voir leur intérêt à se regrouper par secteurs d'activités au sein d'organismes de représentation à portée régionale ou nationale, pour assurer un développement concerté de leurs activités et créer un pont avec les autres secteurs d'activités et avec les diverses instances du milieu touristique.

En travaillant ensemble au sein de l'Association touristique du Nord-du-Québec, Tourisme Baie-James et les représentants des Cris seraient appelés à jouer un rôle actif de représentation des fournisseurs de services afin de tisser un lien entre l'en- semble des intervenants, d'intégrer le produit touristique de la région et de créer une image et une identité fortes de la BaieJames comme destination touristique. En d'autres mots, ils contribueraient à promouvoir ensemble \& l'expérience touristique que peut vivre le visiteur à la Baie James. Cette expérience comporte une combinaison d'activités telles que le tourisme autochtone, nordique, $\mathrm{d}^{5}$ aventure, de grande nature, de chasse et de pêche. En mettant l'accent sur l'expérience touristique globale plutôt que sur un produit spécifique dans leurs activités conjointes de publicité et de promotion, les autorités touristiques de la Baie-James se situeraient dans la lignée de la nouvelle stratégie adoptée en 1998 par Tourisme Québec.

L'enquête réalisée en 1998 auprès des visiteurs de la Baie-James fournit des éléments d'information utiles pour orienter les actions futures des décideurs touristiques. Les visiteurs se disent très satisfaits de leur séjour. Ces visiteurs savent à quoi s'atten-

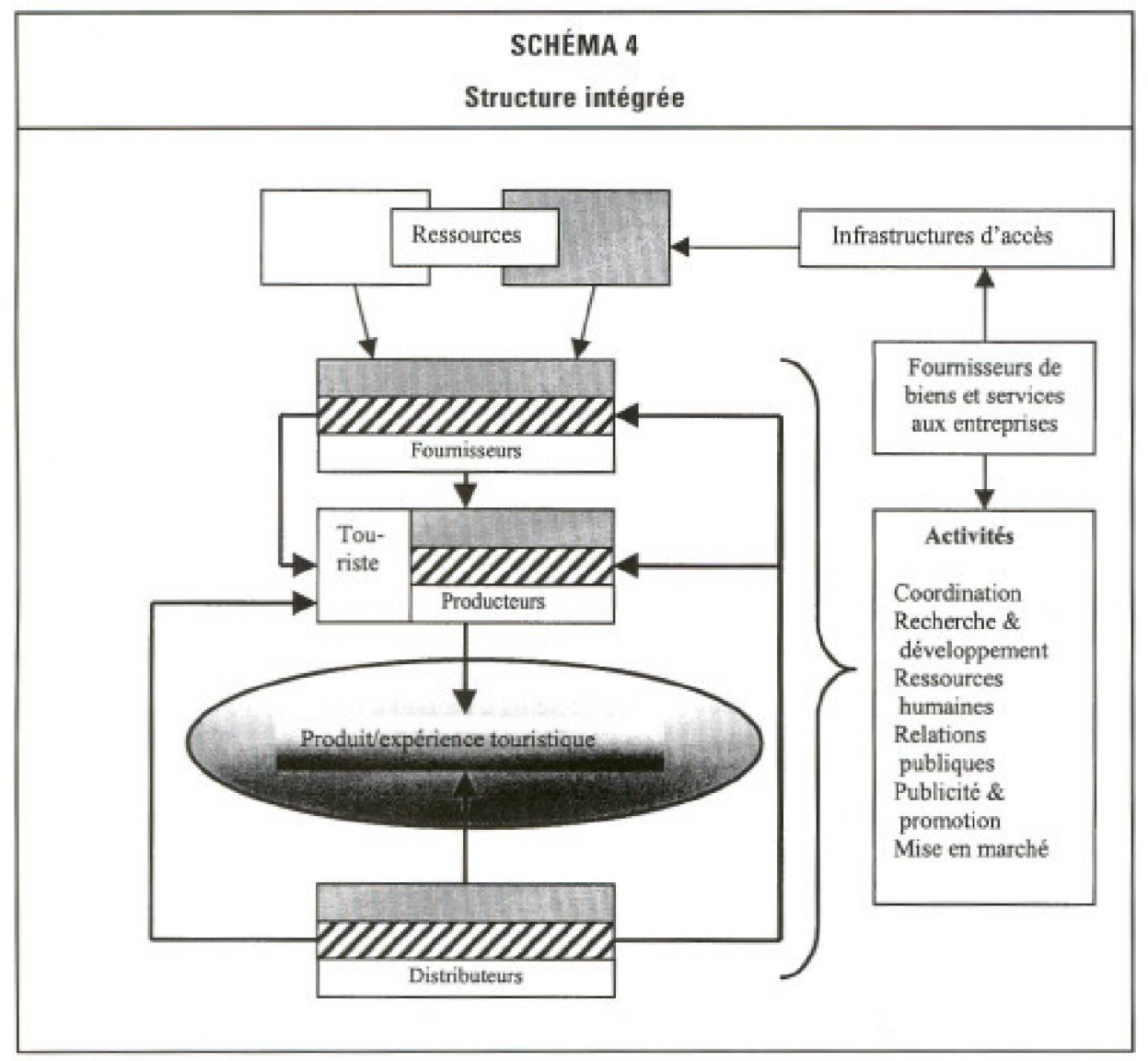

\begin{tabular}{|llll|}
\hline LÉGENDE & Composantes allochtones & Produit/expérience intégré \\
& Composantes autochtones & 77 & Activités communes/purtenariats \\
\hline
\end{tabular}

dre : ils arrivent préparés, équipés, avec leur tente ou en roulotte motorisee. Un client bien informé n'est pas déçu ! Cette clientèle n'est pas nécessairement celle qui dépensera le plus sur le territoire, mais elle permet aux intervenants de la région de prendre le temps de développer et de peaufiner leurs produits, de renforcer l'image et l'identité touristiques de la Baie-James, bref de prendre de l'expérience pour être en mesure de se lancer, le temps venu, sur les marchés internationaux.

\section{CONCLUSION}

Dans le contexte de la mise en place d'une nouvelle Association touristique du Norddu-Québec, le présent article apporte un éclairage théorique sur les efforts de structuration du tourisme a la Baie-James et peut aider les autorités concernées dans leurs prises de décisions. Le concept de grappe industrielle du tourisme, développé en 1993 par un groupe de travail du gouvernement du Québec à partir du modèle des grappes industrielles élaboré par le professeur Michael Porter du Harvard Business School, a servi d'outil d'analyse de 1 industric du tourisme à la Baic-James.

L'analyse fait ressortir l'existence de deux grappes industrielles parallèles du tourisme, l'une autochtone et l'autre allochtone. Le maintien de cette double structure peut constituer un handicap dans la réalisation d'une structuration optimale de l'industrie du tourisme de la Baie-James, particulièrement en ce qui a trait au positionnement et à la mise en marché de l'offre touristique. Les résultats d'une enquête exploratoire menée en 1998 auprès des visiteurs à la Baie James indiquent que la majorité d'entre eux y viennent pour vivre une expérience globale, attirés à la fois par l'offre touristique allochtone et autochtone. Les touristes perçoivent le territoire comme un tout indifferemment des diverses frontières géographiques, ethniques, juridiques ou administratives présentes à la Baie-James. Du point de vue du consommateur, une structure unifiée d'information et de commercialisation de l'offre touristique ou, autrement dit, l"approche du $\alpha$ tout sous le même toit $\%$ est celle qui répondrait le mieux à ses attentes. En ce sens, les objectifs poursuivis par le développement de la nouvelle Association touristique du Nord-du-Québec sont justifiés. 
François Bedard est professeur au Departement d'etudes urbaines et touristiques et chercheur associé à la Chaire de Tourisme de l'Université du Québec à Montréal. Il coordonne, au nom de l'Universite, l'entente de partenariat sur le tourisme entre I'UQAM et la Municipalité de la Baie James ainsi que celle enre I'UQAM et Tourisme Baie-James. Helene Huard est consultante en tourisme. Elle detient un baccalaureat en gestion du tourisme et de l'hôtellevie de I'UOAM.

\section{REMERCIEMENTS}

Les auteurs désirent remercier les personnes suivantes pour leur contribution a la préparation de cet article : Normand Cazelais, Pierre Sénécal et Leon-Marie Hachez d'Hydro-Québec, Stéphane McKenzie de la Municipalité de la Baie James et Lyne l'Africain de Tourisme Baie-James.

Un comité de lecture a lu et accepté ce texte
11 Les éditions de La Griffe de l'Aigle, 1996.

12 Cette enquête, réalisếe en 1998 par HydroQuébec et ses partenaires, avec la collaboration de la Chaire de Tourisme de l'École des Sciences de la gestion de l'UQAM, s'inscrit dans la lignée d'études similaires réalisées depuis 1989 par Hydro-Québec. En tout, 304 questionnaires ont êté traités. Compte tenu de certaines contraintes méthodologiques, les résultats ne constituent qu'une indication partielle du portrait de la clientèle. Une nouvelle étude serait requise pour mieux connaître la clicntèle qui arrive en avion ou en car nolisé pour la visite des ouvrages d'Hydro-Québec ou pour la chasse et la pêche.

13 Commission touristique de la Radissonie (juin 1998), Plan d'action /998-1999.

14 Hydro-Québec, Rappon d'activités 1997, accueil et visites.

15 Selon des observateurs, les deux groupes attirent des clientèles assez différentes, sauf certaines pourvoiries. Par exemple, les Cris recrutent davantage en Ontario et aux ÉtatsUnis que les allochtones.

16 La question des coûts de distribution elevés peut être perçue comme secondaire pour les Cris pour qui l'impératif politique domine ce type de consideration. Selon des observateurs, les Cris ne seraient pas intéressés à développer des services de promotion qui ne seraient pas entièrement contrôlés par leurs propres organismes.

2 Allochtone : terme d'usage à la Baic-James pour désigner les habitants du territoire n'étant pas autochtones, c"est-à-dire n"appartenant pas à la nation crie ou inuit.

3 Le Conseil régional de développement de la Baie James était connu jusqu"en 1999 sous le nom de Conseil régional de la Radissonie.

4 Groupe de travail sur les orientations stratégiques de la grappe industrielle du tourisme (1993), La grappe industrielle du tourisme: proposition d'orienations stratégiques. Les auteurs de cet article se réfèrent à ce modèle non pas pour en faire la promotion, mais uniquement comme cadre d'analyse et de réflexion.

5 Tourisme Québec (1998), Pour donner au monde le gout du Québec, polinque de developpement touristique.

6 La population d'Eastmain compte majoritairement des Cris et une minorité formée d'Inuit.

7 Kunjjuarapik est une communauté inuit:

8 Commission touristique de la Radissonie (1998), Plan d'action 1998-1999, juin.

9 Conseil régional Radissonic (1996), Plan stratégique de developpement, juin.

10 Commission touristique de la Radissonie (1998), Plan d'action 1998-J999, juin.

\section{BIBLIOGRAPHIE}

(1996), Le Québec aurochtone, Éditions La Griffe de l'Aigle.

(1998), * L'industrie touristique autochtone *; Téoros, 17.2.

Association touristique régionale (ATR) (1993), Proposition présentée aux membres de la cortference des municipalités nordiques, Comité régional pour l'implantation d'une association touristique du territoire de la Baie James (CRIAT), mars.

Belanger, L. (1993), Retrospective et bilan de la tournée régionale de consulation sur la création de l'Association touristique du territoire de la baie James, pour le CRIAT, janvier.

Choquette, C. (Gestion Conseil J.P. Corbeil Ine.) et J. Moise (Les Consultants Unaang) (1996), Stratégie de développement du tourime en Radissomie, Société de Développement Touristique, décembre.

Comité de relance de Radisson, Municipalité de la Baie James, localité de Radisson (1995). Plan stratégique de développement de la localité de Radisson 1995-2000, juin.
Commission touristique de la Radissonnie (1998), Plan d'action 1998-1999, juin.

Conseil régional de Radissonie (1996), Plan stratégique de développenent, juin.

Conseil régional de Radissonie (1995), Création d'une Commission regionale du tourisme,

CSN - UQAM (s.d.), Les intervenants towrisiques au Québec, Éditions Téoros.

Fraser, R. (1995), Baie-James: Le guide touristique,

Gouvernement du Québec, Tourisme Québec (1998), Pour donner au monde le goat du Québec, politique de developpement touristique.

Hydro-Québec (1997), Rapport d'activités 1997, accueil et visites.

Hydro-Québec, Etudes sur la fréquentation de la route de la Baie Janes à des fins récréotourixtiques, (1989-1992).

Latraverse S. (Econotech inc) (1994). Etude sur lanalyse des problematiques régionales, région administrative du Nord-du-Quebec, septembre.

Ministère du Tourisme du Québec (1993), Création d'un organisme à vocation towristique dans la portion allochtone du territoire de la Bate James, juillet.

Municipalité de la Baie James (s,d.), L'avenure d'une municipalité urique en son genre.

Office de planification et de développement du Québec, La Direction régionale du Nord-duQuébec (1991), Bilan socio-économique J990, région Nord-du-Quebec.

Worreth, C. (1994), Propositions d'orientations pour la mise en marché des produits touristiques du territoire de la Radissonie, juin.

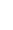

